\title{
Delays in presentation and mortality among Black patients with mechanical thrombectomy after large-vessel stroke at a US hospital
}

\author{
Joshua S. Catapano, MD, Kavelin Rumalla, MD, Visish M. Srinivasan, MD, \\ Candice L. Nguyen, BS, Dara S. Farhadi, MS, Brandon Ngo, BS, Caleb Rutledge, MD, \\ Redi Rahmani, MD, Jacob F. Baranoski, MD, Tyler S. Cole, MD, Ashutosh P. Jadhav, MD, PhD, \\ Andrew F. Ducruet, MD, and Felipe C. Albuquerque, MD \\ Department of Neurosurgery, Barrow Neurological Institute, St. Joseph's Hospital and Medical Center, Phoenix, Arizona
}

OBJECTIVE The incidence and severity of stroke are disproportionately greater among Black patients. In this study, the authors sought to examine clinical outcomes among Black versus White patients after mechanical thrombectomy for stroke at a single US institution.

METHODS All patients who underwent mechanical thrombectomy at a single center from January 1, 2014, through March 31, 2020, were retrospectively analyzed. Patients were grouped based on race, and demographic characteristics, preexisting conditions, clinical presentation, treatment, and stroke outcomes were compared. The association of race with mortality was analyzed in multivariable logistic regression analysis adjusted for potential confounders.

RESULTS In total, 401 patients (233 males) with a reported race of Black $(n=28)$ or White $(n=373)$ underwent mechanical thrombectomy during the study period. Tobacco use was more prevalent among Black patients $(43 \%$ vs $24 \%$, $p=0.04)$, but there were no significant differences between the groups with respect to insurance, coronary artery disease, diabetes, illicit drug use, hypertension, or hyperlipidemia. The mean time from stroke onset to hospital presentation was significantly greater among Black patients (604.6 vs 333.4 minutes) $(p=0.007)$. There were no differences in fluoroscopy time, procedural success (Thrombolysis in Cerebral Infarction grade $2 b$ or 3 ), hospital length of stay, or prevalence of hemicraniectomy. In multivariable analysis, Black race was strongly associated with higher mortality $(32.1 \%$ vs $14.5 \%, p=0.01)$. The disparity in mortality rates resolved after adjusting for the average time from stroke onset to presentation $(p=0.14)$.

CONCLUSIONS Black race was associated with an increased risk of death after mechanical thrombectomy for stroke. The increased risk may be associated with access-related factors, including delayed presentation to stroke centers.

https://thejns.org/doi/abs/10.3171/2021.4.FOCUS2182

KEYWORDS Black patients; large-vessel occlusion; thrombectomy; stroke

$\mathrm{S}$ TROKE, the fifth leading cause of death in the United States, has disproportionately affected Black Americans with respect to incidence and severity, for more than 50 years. ${ }^{1-17}$ According to the REGARDS (Reasons for Geographic and Racial Differences in Stroke) study, only $40 \%$ of the disparity in outcomes between Black and White patients is attributable to differences in traditional stroke risk factors, and the reasons for the remaining disparity are unclear. ${ }^{18}$
Modern mechanical thrombectomy is the gold-standard treatment for acute ischemic stroke caused by largevessel occlusions. ${ }^{19-25}$ During the past decade, landmark randomized trials have demonstrated that mechanical thrombectomy is superior to intravenous alteplase alone when performed within 6 hours after onset of stroke symptoms. ${ }^{26}$ The DAWN (DWI or CTP Assessment With Clinical Mismatch in the Triage of Wake-Up and Late Presenting Strokes Undergoing Neurointervention With

ABBREVIATIONS mRS = modified Rankin Scale; NIHSS = National Institutes of Health Stroke Scale; $\mathrm{TICl}=$ Thrombolysis in Cerebral Infarction .

SUBMITTED February 3, 2021. ACCEPTED April 6, 2021.

INCLUDE WHEN CITING DOI: 10.3171/2021.4.FOCUS2182. 
Trevo) trial recently demonstrated an improvement in 90day disability for patients treated within 6 to 24 hours after stroke onset who had a mismatch between clinical deficit and infarct. ${ }^{27}$

Several studies have demonstrated a racial disparity in the use of thrombectomy and outcomes among patients with acute stroke after an emergent large-vessel occlusion. ${ }^{1,28,29}$ The identification and correction of the sources of this disparity are essential. In this study, we compared stroke outcomes between Black and White patients who underwent mechanical thrombectomy for a stroke at our institution. Furthermore, we investigated potential causes for the disparity in use and outcomes described in earlier literature.

\section{Methods \\ Study Population}

After institutional review board approval, a retrospective cohort study was performed of patients presenting to St. Joseph's Hospital and Medical Center in Phoenix, Arizona. Because of the retrospective nature of the study, the need for patient consent was waived. The study population queried was from an institutional data set of all patients who underwent mechanical thrombectomy for acute ischemic stroke at Barrow Neurological Institute (Phoenix, AZ) from January 1, 2014, to March 31, 2020.

\section{Variables}

The primary predictor variable of interest was race (Black and White). The data set was also reviewed for demographic characteristics (age, sex, and insurance payer status), preexisting conditions (including tobacco, illicit drug, and alcohol use), clinical presentation, and treatment characteristics. Severity of illness was defined according to the National Institutes of Health Stroke Scale (NIHSS) and modified Rankin Scale (mRS) scores at hospital admission. Treatment success was defined as a modified Thrombolysis in Cerebral Infarction (TICI) grade of $2 \mathrm{~b}$ or 3. The data set was reviewed for time from the stroke to admission, time from admission to puncture, and time from admission to revascularization. Procedure time and fluoroscopy time were also calculated. Clinical outcomes of interest included the hospital length of stay, mortality, NIHSS score, and the mRS score (poor outcome defined as an $\mathrm{mRS}$ score $>2$ ) at the last follow-up.

\section{Statistical Analysis}

IBM SPSS version 27 (IBM Corp.) was used for all analyses. Statistical significance was set at $p<0.05$. Frequencies and descriptive statistics were used to describe the study population. Means were reported with standard deviations (SDs). Univariate analysis was conducted to compare demographic characteristics, preexisting conditions, clinical presentation, treatment characteristics, and outcomes for Black and White patients. Nonparametric tests were used for the univariate analysis. The interval data did not meet the assumptions of normality per Shapiro-Wilk and Q-Q plots. A multivariate analysis was conducted to study the independent relationship between patient race and mortality. Covariates in the mortality model were selected on the basis of clinical suspicion and significance in univariate analysis. A multilevel (hierarchical) binary logistic regression model was employed to adjust for confounders and identify meaningful effect modifiers on the causative pathway between race and stroke outcomes. Here, "confounder" was defined as a factor associated with both the exposure and outcome that does not lie on the causative pathway. By contrast, a variable defined as an effect modifier does lie on the causative pathway and deserves attention.

\section{Results \\ Description of Cohort}

Of 565 patients who underwent a mechanical thrombectomy during the study period, race was recorded for 525 , with 401 patients identified as either Black or White; 28 were Black $(7.0 \%)$ and 373 were White $(93.0 \%)$. The mean age was 68.3 years (SD 13.5 years), and 233 (58.1\%) of these patients were men. The mean NIHSS score was 15.2 (SD 7.5) at admission, and 176 patients (43.9\%) received intravenous alteplase. The majority of patients had successful revascularization with TICI grades of $2 \mathrm{~b}$ or 3 $(\mathrm{n}=340,84.8 \%)$, and the mean door-to-puncture time was 50.5 minutes (SD 49.6 minutes). Hemicraniectomy was required in 12 patients $(3.0 \%)$. The rate of hemorrhagic conversion with symptomatic intracerebral hemorrhage was $1.7 \%$ (7 of 401 patients).

The mean follow-up time was 117 days (SD 309 days). At follow-up, 278 patients (69.3\%) had mRS scores $>2$ and 63 patients (15.7\%) had died. A comparison of the demographic characteristics, preexisting conditions, clinical presentation, treatment characteristics, and outcomes of Black patients and White patients is reported in Tables $1-3$.

\section{Demographics and Preexisting Conditions}

White patients had a higher mean age (68.9 years [SD 13.2 years]) compared with Black patients (61.1 years [SD 13.5 years] $)(p=0.01)$. Smoking was more prevalent among Black patients than White patients (12 [42.9\%] vs 90 [24.1\%], $\mathrm{p}=0.03$ ); however, there were no other statistically significant differences between racial groups with respect to sex, BMI, prior stroke, current use of antiplatelets or anticoagulants, insurance status, coronary artery disease, diabetes, hypertension, or hyperlipidemia ( $\mathrm{p}>$ 0.05 for all).

\section{Clinical Presentation and Treatment Characteristics}

The average NIHSS score at admission was similar between the two racial groups. However, the mean time from stroke onset to hospital presentation was significantly greater for Black patients (604.6 minutes) than White patients (333.4 minutes) ( $\mathrm{p}=0.007)$. At our institution, the mean time from presentation to revascularization was shorter for Black patients (66.8 minutes) than White patients (93.5 minutes) $(\mathrm{p}=0.04)$. Total procedural time was significantly shorter for Black patients (27 minutes) than for White patients (43 minutes) $(\mathrm{p}=0.002)$. There were no statistically significant differences in fluoroscopy time, procedural success (TICI grade $2 \mathrm{~b}$ or 3 ), hospital length 
TABLE 1. Comparison of demographic characteristics and preexisting conditions

\begin{tabular}{lcccc}
\hline \multicolumn{1}{c}{ Variable } & All Patients $(n=401)$ & Black Patients $(n=28)$ & White Patients $(n=373)$ & $p$ Value \\
\hline Mean age, yrs (SD) & $68.3(13.5)$ & $61.1(15.4)$ & $68.9(13.2)$ & 0.01 \\
\hline Male sex & $233(58.1)$ & $21(75.0)$ & $212(56.8)$ & 0.06 \\
\hline Insurance & & & $300(80.4)$ & 0.16 \\
\hline Medicare/Medicaid & $318(79.3)$ & $18(64.3)$ & $22(5.9)$ & \\
\hline Commercial & $26(6.5)$ & $4(14.3)$ & $37(10.0)$ & \\
\hline None & $42(10.5)$ & $5(17.9)$ & $14(3.8)$ & \\
\hline Other & $15(3.7)$ & $1(3.6)$ & $29.0(6.3)$ & 0.61 \\
\hline Mean BMI (SD) & $29.1(6.4)$ & $30.2(7.8)$ & $68(18.2)$ & 0.60 \\
\hline Prior stroke & $72(18.0)$ & $4(14.3)$ & $90(24.1)$ & 0.03 \\
\hline Tobacco smoking & $102(25.4)$ & $12(42.9)$ & $157(42.1)$ & 0.28 \\
\hline HTN & $293(73.1)$ & $18(64.3)$ & $109(29.2)$ & 0.94 \\
\hline CAD & $168(41.9)$ & $11(39.3)$ & $146(39.1)$ & 0.06 \\
\hline DM & $117(29.2)$ & $8(28.6)$ & $73(19.6)$ & 0.83 \\
\hline HLD & $162(40.4)$ & $16(57.1)$ & $116(31.1)$ & 0.61 \\
\hline Anticoagulation use & $78(19.5)$ & $5(17.9)$ & $10(35.7)$ & \\
\hline Antiplatelet use & $126(31.4)$ & & & \\
\hline
\end{tabular}

$C A D=$ coronary artery disease; $D M=$ diabetes mellitus; $H L D=$ hyperlipidemia; $H T N=$ hypertension .

Values represent the number of patients (\%) unless stated otherwise.

TABLE 2. Comparison of clinical presentation and treatment characteristics

\begin{tabular}{|c|c|c|c|c|}
\hline Variable & All Patients $(n=401)$ & Black Patients $(n=28)$ & White Patients $(n=373)$ & p Value \\
\hline Mean stroke onset to presentation, mins (SD) & $353.2(484)$ & $604.6(805.2)$ & $333.4(445.8)$ & 0.007 \\
\hline Mean NIHSS score at admission (SD) & $15.2(7.5)$ & $15.2(8.2)$ & $15.1(7.5)$ & 0.40 \\
\hline Median mRS score at admission (25th-75th percentile) & $4(3-5)$ & $4(2-5)$ & $4(3-5)$ & 0.38 \\
\hline Vessel occlusion, $\mathrm{n}(\%)$ & & & & 0.39 \\
\hline MCA & $205(51.1)$ & $14(50)$ & $191(51)$ & \\
\hline MCA/ICA & $58(14.5)$ & $7(25.0)$ & $51(13.7)$ & \\
\hline Cervical ICA & $48(12.0)$ & $3(10.7)$ & $45(12.1)$ & \\
\hline Intracranial ICA & $32(8.0)$ & $2(7.1)$ & $30(8.1)$ & \\
\hline Tandem ICA & $18(4.5)$ & $0(0.0)$ & $18(4.9)$ & \\
\hline Basilar & $27(6.7)$ & $0(0.0)$ & $27(7.3)$ & \\
\hline ACA/ICA & $5(1.2)$ & $1(3.6)$ & $4(1.1)$ & \\
\hline Other & $8(2.0)$ & $1(3.6)$ & $7(1.9)$ & \\
\hline IV tPA, n (\%) & $176(43.9)$ & $9(32.1)$ & $167(44.8)$ & 0.19 \\
\hline sICH, n (\%) & $7(1.7)$ & $0(0.0)$ & $7(1.9)$ & * \\
\hline Mean admission to puncture, mins (SD) & $50.5(49.6)$ & $38.6(40.5)$ & $51.3(50.1)$ & 0.30 \\
\hline Mean admission to revascularization, mins (SD) & $91.8(65.7)$ & $66.8(57.7)$ & $93.5(65.9)$ & 0.04 \\
\hline Mean procedure time, mins (SD) & $42.0(29.3)$ & $27.3(27.0)$ & $43.0(29.2)$ & 0.002 \\
\hline Mean fluoroscopy time, mins (SD) & $23.2(15.3)$ & $24.0(22.0)$ & $23.2(14.6)$ & 0.59 \\
\hline $\mathrm{TICl}$ grade $2 \mathrm{~b} / 3, \mathrm{n}(\%)$ & $340(84.8)$ & 23 (82.1) & $317(85.0)$ & 0.63 \\
\hline
\end{tabular}

$\mathrm{ACA}=$ anterior cerebral artery; ICA = internal carotid artery; IV tPA = intravenous tissue plasminogen activator; $\mathrm{MCA}=$ middle cerebral artery; sICH = symptomatic intracerebral hemorrhage.

* Unable to calculate $p$ value due to insufficient sample size for comparative groups. 
TABLE 3. Comparison of clinical outcomes

\begin{tabular}{lccrc}
\hline \multicolumn{1}{c}{ Variable } & All Patients $(\mathrm{n}=401)$ & Black Patients $(\mathrm{n}=28)$ & White Patients $(\mathrm{n}=373)$ & $\mathrm{p}$ Value \\
\hline Mean length of stay, days (SD) & $8.5(8.2)$ & $7.2(4.9)$ & $8.6(8.4)$ & 0.89 \\
\hline Hemicraniectomy, $\mathrm{n}(\%)$ & $12(3.0)$ & $1(3.6)$ & $11(2.9)$ & 0.85 \\
\hline Mortality, $\mathrm{n}(\%)$ & $63(15.7)$ & $9(32.1)$ & $54(14.5)$ & 0.01 \\
\hline mRS score >2, $\mathrm{n}(\%)$ & $278(69.3)$ & $23(82.1)$ & $255(68.4)$ & 0.13 \\
\hline Mean NIHSS score at discharge (SD) & $11.1(10.9)$ & $15.2(13.6)$ & $10.8(10.7)$ & 0.14 \\
\hline Mean NIHSS score at last follow-up (SD) & $13.2(14.0)$ & $17.6(14.5)$ & $12.9(13.9)$ & 0.02 \\
\hline Median mRS score at last follow-up (25th- & $4(2-5)$ & $4(3-5)$ & $4(2-5)$ & 0.11 \\
75th percentile) & & & & \\
\hline
\end{tabular}

of stay, or rate of hemicraniectomy (Tables 2 and 3; p > 0.05 for all).

\section{Clinical Outcomes}

At follow-up, Black patients had a higher risk of mortality following mechanical thrombectomy than White patients $(9$ [32.1\%] vs 54 [14.5\%], $\mathrm{p}=0.01)$. The mean NIHSS score was also significantly greater at the last follow-up for Black patients (17.6 [SD 14.5] vs 12.9 [SD 13.9], $\mathrm{p}=0.02$ ).

\section{Hierarchical Binary Logistic Regression}

Hierarchical binary logistic regression analysis was used to analyze the relationship between patient race and stroke mortality. The analysis isolated "time from stroke onset to presentation" as a major effect modifier on the causative pathway between patient race and stroke mortality. Before addition of this effect modifier, Black race was a significant predictor in the binary logistic regression analysis (OR 4.44, 95\% CI 1.37-14.41; $\mathrm{p}=0.01$ ). After the addition of "time from stroke onset to presentation," Black race was no longer a predictor of mortality (OR 2.62, 95\% CI 0.74-9.24; $\mathrm{p}=0.14)$. Other covariates that were adjusted for included age, sex, smoking status, insurance status, NIHSS score, door-to-puncture time, procedure time, and TICI grade. These covariates were not associated with stroke mortality and did not influence the effect of patient race on stroke mortality (all $p>0.25$ ). The hierarchical models with and without "time from stroke onset to presentation" are presented in Table 4.

\section{Discussion}

In this single-center comparison of outcomes after mechanical thrombectomy, we found higher rates of mortality and longer times to presentation in Black patients than in White patients. Inequalities in stroke severity and mortality between Black patients and White patients are extensively reported in earlier literature. ${ }^{15}$ This inequality was demonstrated in a multivariable analysis that was adjusted for potential confounders in age, sex, insurance status, preexisting conditions, smoking status, NIHSS score at admission, door-to-puncture time, and TICI grade. Furthermore, patients in both racial groups were similar with respect to demographic conditions, preexisting conditions, and presenting stroke severity (NIHSS score). All patients at our institution had comparable rates of successful recanalization, with faster door-to-revascularization times in Black patients. However, recent nationwide studies have demonstrated racial and ethnic disparities in the use of mechanical thrombectomy for stroke., ${ }^{1,28,29}$ These studies acknowledge that the disparity in use of mechanical thrombectomy

TABLE 4. Hierarchical binary logistic regression model for stroke mortality

\begin{tabular}{|c|c|c|c|c|}
\hline & \multicolumn{2}{|c|}{ Before Addition of Effect Modifier } & \multicolumn{2}{|c|}{ After Addition of Effect Modifier } \\
\hline & $\mathrm{OR}(95 \% \mathrm{Cl})$ & p Value & OR $(95 \% \mathrm{Cl})$ & $p$ Value \\
\hline \multicolumn{5}{|l|}{ Variable } \\
\hline Race & $4.4(1.4-14.4)$ & 0.01 & $2.6(0.7-9.2)$ & 0.14 \\
\hline Age, per yr & $1.0(0.9-1.0)$ & 0.28 & $1.0(1.0-1.0)$ & 0.70 \\
\hline Male sex & $0.8(0.4-1.6)$ & 0.59 & $0.8(0.4-1.6)$ & 0.50 \\
\hline \multicolumn{5}{|l|}{ Insurance } \\
\hline Medicaid/Medicare & Reference & & Reference & \\
\hline Commercial & $1.2(0.5-2.9)$ & 0.59 & $1.6(0.6-4.4)$ & 0.35 \\
\hline Tobacco smoking & $1.4(0.6-2.9)$ & 0.43 & $2.0(0.9-4.5)$ & 0.09 \\
\hline Admission NIHSS score & $1.0(0.9-1.0)$ & 0.64 & $1.0(0.9-1.0)$ & 0.75 \\
\hline Door-to-puncture, mins & $1.0(1.0-1.0)$ & 0.42 & $1.0(1.0-1.0)$ & 0.33 \\
\hline Procedure time, mins & $1.0(1.0-1.0)$ & 0.68 & $1.0(1.0-1.0)$ & 0.91 \\
\hline $\mathrm{TICl}$ grade $2 \mathrm{~b} / 3$ & $1.2(0.4-3.8)$ & 0.71 & $1.0(0.3-3.0)$ & 0.96 \\
\hline
\end{tabular}


may be attributed to preadmission factors, such as delayed time from stroke onset to hospital presentation.

Our results show that the mean time from stroke onset to hospital presentation for Black patients was almost twice that for White patients. Delayed presentations lead to an increased size of the ischemic core and a smaller radius of the salvageable penumbra..$^{30}$ This undoubtedly decreases the overall use and efficacy of mechanical thrombectomy; however, the present study did not analyze the size of the ischemic core or the radius of the penumbra. The cause of delayed presentation among Black patients is multifactorial. Earlier literature suggested that delayed presentation may be due to disparities in acute stroke treatment allocation, emergency medical service use, and personal health education, as well as the exclusion of predominantly Black neighborhoods from mainstream resources. ${ }^{16}$ Furthermore, in a cohort of patients treated for stroke at a tertiary-care hospital, Springer et al. found that Black patients were less likely than White patients to use emergency medical services and were more likely to have delayed hospital arrival times ( $\geq 3$ hours) after stroke onset. ${ }^{31}$ In 2 earlier nationwide analyses, Attenello et al. ${ }^{1}$ and Brinjikji et al. ${ }^{28}$ both found that Black patients had significantly fewer admissions to hospitals performing mechanical thrombectomy procedures at high volumes. Black patients were more likely than White patients to be admitted to low-resource centers without endovascular capabilities and subsequently transferred to stroke centers. Brinjikji et al. demonstrated that racial group but not insurance status was associated with disparities in access to mechanical thrombectomy. ${ }^{28}$ In a population-based study in southern Texas, Smith et al. found that Hispanic patients were less likely than non-Hispanic patients to arrive at stroke centers by emergency medical services. ${ }^{32}$ The disparity of thrombectomy access and presentation timing in ethnic groups might be mitigated by institutional solutions, including stroke education targeted to groups associated with disparities and clinics and/or emergency departments in underprivileged areas for more accessible healthcare access.

Despite similar NIHSS scores at hospital admission and discharge, Black patients had significantly higher mean NIHSS scores at long-term follow-up. In earlier literature, it has been reported that Black patients experienced more long-term disability after stroke than White patients. ${ }^{33}$ This finding is attributed to multiple factors, including disparities in access to rehabilitation, primary care follow-up, and social support. In the present study, descriptive analysis of effect sizes for several other functional outcomes, including an mRS score $>2$ at the latest follow-up, also indicated racial disparities. We suspect these clinically important factors represent type II errors, and future studies with larger sample sizes should be conducted.

\section{Limitations}

The retrospective and single-center study design inherently limits the generalizability of our results. Black patients were a significant minority, comprising only $7 \%$ of the cohort (28 patients). The small sample size weakened our statistical power and is an acknowledged limitation of the study. When interpreting results, we recommend examining effect sizes, frequencies, and means in the context of clinical judgment and experience. We encourage the design of larger multi-institutional studies to analyze this critical healthcare disparity further. Our study did not analyze the size of the ischemic core or the radius of the penumbra. The reasons for delayed presentation in Black patients are unknown and warrant investigation in future studies and quality-improvement efforts.

\section{Conclusions}

Compared with White patients, Black patients in our cohort had a higher risk of death following mechanical thrombectomy for stroke. Black patients had nearly twice the average time from stroke symptom onset to hospital presentation. The increased risk appeared to be associated with access-related factors. Hierarchical logistic regression analysis revealed that delayed presentation was a significant effect modifier in the causative pathway between patient race and stroke mortality. Further research and improvements are needed to optimize the social determinants of health among patients who experience stroke.

\section{Acknowledgments}

We thank the staff of Neuroscience Publications at Barrow Neurological Institute for assistance with manuscript preparation.

\section{References}

1. Attenello FJ, Adamczyk P, Wen G, et al. Racial and socioeconomic disparities in access to mechanical revascularization procedures for acute ischemic stroke. J Stroke Cerebrovasc Dis. 2014;23(2):327-334.

2. Ayala C, Croft JB, Greenlund KJ, et al. Sex differences in US mortality rates for stroke and stroke subtypes by race/ethnicity and age, 1995-1998. Stroke. 2002;33(5):1197-1201.

3. Ayala C, Greenlund KJ, Croft JB, et al. Racial/ethnic disparities in mortality by stroke subtype in the United States, 19951998. Am J Epidemiol. 2001;154(11):1057-1063.

4. Bian J, Oddone EZ, Samsa GP, et al. Racial differences in survival post cerebral infarction among the elderly. Neurology. 2003;60(2):285-290.

5. Broderick J, Brott T, Kothari R, et al. The Greater Cincinnati/Northern Kentucky Stroke Study: preliminary first-ever and total incidence rates of stroke among blacks. Stroke. 1998;29(2):415-421.

6. Fullerton HJ, Chetkovich DM, Wu YW, et al. Deaths from stroke in US children, 1979 to 1998. Neurology. 2002;59(1): 34-39.

7. Gillum RF, Mussolino ME. Education, poverty, and stroke incidence in whites and blacks: the NHANES I Epidemiologic Follow-up Study. J Clin Epidemiol. 2003;56(2): $188-195$.

8. Howard G, Howard VJ, Katholi C, et al. Decline in US stroke mortality: an analysis of temporal patterns by sex, race, and geographic region. Stroke. 2001;32(10):2213-2220.

9. Johnson NE. The racial crossover in comorbidity, disability, and mortality. Demography. 2000;37(3):267-283.

10. Jones MR, Horner RD, Edwards LJ, et al. Racial variation in initial stroke severity. Stroke. 2000;31(3):563-567.

11. Kissela B, Broderick J, Woo D, et al. Greater Cincinnati/ Northern Kentucky Stroke Study: volume of first-ever ischemic stroke among blacks in a population-based study. Stroke. 2001;32(6):1285-1290. 
12. Kissela B, Schneider A, Kleindorfer D, et al. Stroke in a biracial population: the excess burden of stroke among blacks. Stroke. 2004:35(2):426-431.

13. Kuhlemeier KV, Stiens SA. Racial disparities in severity of cerebrovascular events. Stroke. 1994;25(11):2126-2131.

14. Muntner P, Garrett E, Klag MJ, Coresh J. Trends in stroke prevalence between 1973 and 1991 in the US population 25 to 74 years of age. Stroke. 2002;33(5):1209-1213.

15. Stansbury JP, Jia H, Williams LS, et al. Ethnic disparities in stroke: epidemiology, acute care, and postacute outcomes. Stroke. 2005;36(2):374-386.

16. Williams O, Ovbiagele B. Stroking out while black-the complex role of racism. JAMA Neurol. 2020;77(11):13431344.

17. Worrall BB, Johnston KC, Kongable G, et al. Stroke risk factor profiles in African American women: an interim report from the African-American Antiplatelet Stroke Prevention Study. Stroke. 2002;33(4):913-919.

18. Howard G, Moy CS, Howard VJ, et al. Where to focus efforts to reduce the black-white disparity in stroke mortality: incidence versus case fatality? Stroke. 2016;47(7):1893-1898.

19. Powers WJ, Rabinstein AA, Ackerson T, et al. Guidelines for the early management of patients with acute ischemic stroke: 2019 update to the 2018 guidelines for the early management of acute ischemic stroke: a guideline for healthcare professionals From the American Heart Association/American Stroke Association. Stroke. 2019;50(12):e344-e418.

20. Mokin M, Ansari SA, McTaggart RA, et al. Indications for thrombectomy in acute ischemic stroke from emergent large vessel occlusion (ELVO): report of the SNIS Standards and Guidelines Committee. J Neurointerv Surg. 2019;11(3): 215-220.

21. Palaniswami M, Yan B. Mechanical thrombectomy is now the gold standard for acute ischemic stroke: implications for routine clinical practice. Intervent Neurol. 2015;4(1-2):18-29.

22. Boisseau W, Escalard S, Fahed R, et al. Direct aspiration stroke thrombectomy: a comprehensive review. J Neurointerv Surg. 2020;12(11):1099-1106.

23. Kuybu O, Javalkar V, Amireh A, et al. Implications of the use of mechanical thrombectomy on outcome in large vessel occlusion following the 2015 landmark trials. J Neurointerv Surg. 2021;13(1):4-7.

24. Fredrickson VL, Bonney PA, Rangwala SD, et al. Comparison of aspiration-first versus stentriever-first techniques in performing mechanical thrombectomy for large vessel occlusions. J Neurointerv Surg. 2021;13(7):614-618.

25. Yaeger KA, Martini ML, Hardigan T, et al. Mortality reduction after thrombectomy for acute intracranial large vessel occlusion: meta-analysis of randomized trials. J Neurointerv Surg. 2020;12(6):568-573.
26. Goyal M, Menon BK, van Zwam WH, et al. Endovascular thrombectomy after large-vessel ischaemic stroke: a metaanalysis of individual patient data from five randomised trials. Lancet. 2016;387(10029):1723-1731.

27. Nogueira RG, Jadhav AP, Haussen DC, et al. Thrombectomy 6 to 24 hours after stroke with a mismatch between deficit and infarct. N Engl J Med. 2018;378(1):11-21.

28. Brinjikji W, Rabinstein AA, Cloft HJ. Socioeconomic disparities in the utilization of mechanical thrombectomy for acute ischemic stroke. J Stroke Cerebrovasc Dis. 2014;23(5): 979-984.

29. Rinaldo L, Rabinstein AA, Cloft H, et al. Racial and ethnic disparities in the utilization of thrombectomy for acute stroke. Stroke. 2019;50(9):2428-2432.

30. Gomez CR. Editorial: Time is brain! J Stroke Cerebrovasc Dis. 1993;3(1):1-2.

31. Springer MV, Labovitz DL, Hochheiser EC. Race-ethnic disparities in hospital arrival time after ischemic stroke. Ethn Dis. 2017;27(2):125-132.

32. Smith MA, Lisabeth LD, Bonikowski F, Morgenstern LB. The role of ethnicity, sex, and language on delay to hospital arrival for acute ischemic stroke. Stroke. 2010;41(5):905-909.

33. Faigle R, Cooper LA. Explaining and addressing racial disparities in stroke care and outcomes: a puzzle to solve now. Neurology. 2019;93(18):773-775.

\section{Disclosures}

The authors report no conflict of interest concerning the materials or methods used in this study or the findings specified in this paper.

\section{Author Contributions}

Conception and design: Albuquerque, Catapano, Jadhav, Ducruet. Acquisition of data: Nguyen, Farhadi, Ngo, Rutledge, Rahmani, Baranoski. Analysis and interpretation of data: Catapano, Rumalla, Srinivasan. Drafting the article: Catapano, Rumalla. Critically revising the article: Albuquerque, Srinivasan, Rutledge, Rahmani, Baranoski, Cole, Jadhav, Ducruet. Reviewed submitted version of manuscript: Albuquerque. Statistical analysis: Catapano, Rumalla, Cole. Study supervision: Albuquerque.

\section{Correspondence}

Felipe C. Albuquerque: c/o Neuroscience Publications, Barrow Neurological Institute, St. Joseph's Hospital and Medical Center, Phoenix, AZ.neuropub@barrowneuro.org. 